\title{
Transgenic Mice Expressing Luciferase Under a 4.5 kb Tyrosine Hydroxylase Promoter
}

Kenneth W.. Dodd, Terry C.. Burns, Stephen M.. Wiesner, Elina Kudishevich, Dominic T.. Schomberg, Bo-Woo Jung, Ji-Eun Kim, John R.. Ohlfest, Walter C.. Low

1.

Corresponding author: Kenneth W.. Dodd, dodd0035@umn.edu

\section{Abstract}

Background: Animal models have provided an important tool for the study of neurological disorders, such as Parkinson's disease. To date, however, no high throughput techniques have been available for the specific visualization of catecholaminergic neurons in vivo.

Methods: Here, we generated transgenic mice with a $4.5 \mathrm{~kb}$ tyrosine hydroxylase promoter driving a firefly luciferase reporter gene by pronuclear injection.

Results: Though variations were observed between generated lines, those selected demonstrated bioluminescence in the olfactory bulb, hypothalamus/medial forebrain bundle, locus ceruleus, and ventral midbrain, consistent with known areas of tyrosine hydroxylase expression. Additionally, expression of luciferase was observed in certain non-catecholaminergic brain regions, with overall highest expression observed in the dorsal midbrain.

Conclusions: To our knowledge, these mice represent the first reported animals created with a neurotransmitter system-specific promoter controlling expression of a bioluminescent reporter molecule. This strategy may facilitate future studies of dopaminergic neuron neurodegeneration and neuroprotection.

Categories: Genetics, Miscellaneous, Neurosurgery

Keywords: neuroprotection, neurodegeneration, bioluminescence, animal model, translational research, catecholamines, transgenic mice, tyrosine hydroxylase, firefly luciferase

\section{Introduction}

Tyrosine hydroxylase (TH) catalyzes the first and rate-limiting step in the biosynthesis of catecholamine neurotransmitters: hydroxylation of the amino acid tyrosine into dihydroxyphenylalanine (dopa). From dopa, sequential enzymatic reactions yield dopamine (DA), norepinephrine (NE), and epinephrine (EPI). Together, DA, NE, and EPI make up the major neurotransmitter constituents of catecholaminergic pathways in the central nervous system (CNS). CNS catecholamines play a role in a wide array of functions, such as motor movement, learning, memory, emotion, and sleep. As such, catecholaminergic neurons are implicated

Published 08/15/2011

() Copyright 2011 Dodd et al. This is an open access article distributed under the terms of the Creative Commons Attribution License CC-BY 3.0., which permits unrestricted use, distribution, and reproduction in any medium, provided the original author and source are credited.
In particular, $\mathrm{PD}$ is characterized largely by the gradual loss of TH-expressing dopaminergic neurons in the substantia nigra pars compacta (SNc). Although advances in symptom management, such as levo-dopa therapy [1] and deep brain stimulation [2] have arisen, no treatments currently exist to halt progression of PD or replace lost SNc DA neurons. Stopping the loss of DA neurons in the SNc or transplanting DA neurons into the striatum has been the focus of intensive investigation.

Translational studies for PD treatment often utilize animal models, many of which involve DA neuron loss in the SNc by the actions of selective neurotoxins [3-4]. These in vivo models of PD can be useful when studying neuroprotective compounds [5-6] and cell replacement strategies [7]. The models have also provided the impetus for a number of clinical trials [8]. A key outcome measure in animal studies of neuroprotection and cell replacement is the observation of a relatively high number of DA neurons in treatment groups compared to non-treatment controls.

Conventional approaches to quantitative evaluation of DA neuron numbers over time include immunohistochemistry (IHC) and micro positron emission tomography (microPET); recently, MRI-based techniques have also been described [9-10]. Although microPET and MRI provide detailed anatomical information, they are expensive, time-consuming, and not readily available to most investigators [11]. IHC studies, therefore, represent the current gold standard for anatomic resolution and cell number tabulation. Unfortunately, IHC does not allow for longitudinal investigations of safety and efficacy in individual animals. Rather, each analysis point requires sacrifice of a discrete animal cohort. To date, development of an efficient, economical, and non-invasive technique to quantify the relative number of viable dopaminergic neurons in living animals has remained an elusive goal. 
Bioluminescent reporter systems employing luciferase enzymes have been demonstrated to provide a safe, high-throughput, low-cost, quantifiable serial imaging system of disease processes [12-13]. Luciferases have been shown to produce long-lasting, localizable signals in mammalian cells [14] with the relatively long wavelength of most luciferase-mediated reactions providing robust tissue penetration. These properties have led many groups to use luciferase for tracking cells in vivo, including several studies that have used the enzymes intracranially [15-16].

In this study, we used a firefly luciferase reporter to create transgenic mice with quantifiable bioluminescence in TH-positive neurons. These mice express firefly luciferase transcriptionally regulated by the $4.5 \mathrm{~kb}$ rat $\mathrm{TH}$ promoter. The bioluminescence patterns of these mice include expression patterns previously described using the $4.5 \mathrm{~kb}$ TH promoter to drive placental alkaline phosphatase (TH-AP) [17]. To our knowledge, these TH-Luc mice represent the first transgenic animals with a neurotransmitter systemspecific promoter driving expression of a luciferase reporter gene. This strategy may prove useful to PD research by allowing for bioluminescence-based quantification of relative DA cell numbers in the SNc or in in vitro assays. Furthermore, mice expressing luciferase under DA-specific promoters have potentially valuable applications in dopaminergic cell replacement studies as a source of stem cells or primary DA neurons that can be tracked non-invasively.

\section{Abbreviations}

AP- alkaline phosphatase

CB- cerebellum

CMV- cytomegalovirus

CNS- central nervous system

CTX- cerebral cortex

DA- dopamine

DAT- dopamine transporter

DM- dorsal midbrain

dopa- dihydroxyphenylalanine

EPI- epinephrine

H/MFB- hypothalamus and medial forebrain bundle

HF- hippocampal formation

IHC- immunohistochemistry

LC- locus ceruleus

Luc- luciferase

NE- norepinephrine

OB- olfactory bulbs

PD- Parkinson's disease

ROI- region of interest

SNc- substantia nigra pars compacta

STR- striatum

TH- tyrosine hydroxylase

UTR- untranslated region 


\section{Materials And Methods}

\section{Animals}

All animals were housed and maintained in accordance with the Guide for the Care and Use of Laboratory Animals of the Public Health Service, and guidelines set by the University of Minnesota Institutional Animal Care and Use Committee. They were housed under specific pathogen-free conditions with ad libitum food and water and were exposed to $12 \mathrm{~h} / 12 \mathrm{~h}$ light/dark cycles. Measures were taken to minimize the number of animals used and to avoid animal suffering. Wild type mice were FVB/N, obtained from Jackson Laboratories (Bar Harbor, Maine). Positive control CMV-Luciferase mice were obtained from Xenogen Corporation (Hopkinton, MA).

\section{Generation of TH-Luc transgenic mice}

For construction of the $\mathrm{p} \beta$-globin4.5TH-Luc plasmid (Fig. 1), the $\beta$-globin marginal element from pCpGvitro-neo-mcs G2 (Invivogen Corp, San Diego, CA) was amplified by polymerase chain reaction (PCR). PCR primers were designed to add MluI restriction enzyme sites to both ends of the PCR product. The PCR product was gel purified using the UltraClean GelSpin DNA Purification Kit per the manufacturer's instructions (Mo-Bio Laboratories, Carlsbad, CA) and subcloned into the pCR2.1TOPO TA vector (Invitrogen, Carlsbad, CA). The pCR2.1- $\beta$-globin construct was digested with MluI; resultant fragments were then purified and ligated into the p4.5TH-Luc plasmid that was linearized beforehand by digestion with MluI. The p4.5TH-Luc plasmid (a kind gift from Dona M. Chikaraishi, Duke University) contained the North American firefly (Photinus pyralis) luciferase controlled by a $4.5 \mathrm{~kb}$ rat TH 5' flanking sequence [17].

Plasmid DNA for pronuclear injection was prepared from $\mathrm{p} \beta$-globin4.5TH-Luc by linearization with HpaI. Digested DNA was isolated by agarose gel electrophoresis and visualized using crystal violet. Linear DNA was extracted from the gel as described above. Extracted fragments were precipitated twice with ethanol and then resuspended in injection buffer (5 mM Tris [pH 7.4] and 0.1 mM EDTA). DNA was dialyzed against four liters of injection buffer overnight in a Slide-a-Lyzer Cassette (Pierce, Rockford, IL). Dialyzed DNA was quantified using an UV spectrophotometer, visualized on an agarose gel, and adjusted to a concentration of $2 \mathrm{ng} / \mathrm{ml}$. The DNA was then injected into the pronuclei of FVB/N embryos by the Mouse Genetics Laboratory at the University of Minnesota. Microinjected oocytes were subsequently transferred into the oviducts of pseudopregnant FVB/N foster mothers.

\section{Genotyping and in vivo screening}

Genomic DNA was isolated from the resultant mice by phenol extraction from tail biopsies. DNA concentrations were quantified using a UV spectrophotometer, then $500 \mu \mathrm{g}$ of the isolated DNA was amplified by PCR. PCR primers (TH-Luc sense - ACGCGTGGTTGAACTGCTGATT; TH-Luc antisense CCCAAATGCCAGAGCAATTAGCC) were designed to amplify a $683 \mathrm{bp}$ segment of the transgene encoding the $\beta$-globin marginal element and a portion of the TH firefly luciferase coding sequence. PCR revealed twelve TH-Luc founder lines of mice that carried the transgene. These twelve TH-Luc mouse lines were maintained as heterozygotes on a FVB/N background. The F1 offspring of the 12 founder lines were again screened by PCR to verify germline transmission.

Transgene presence was functionally assessed by bioluminescence imaging with a Xenogen IVIS 50 Imaging System. F1 offspring of the 12 founder lines were sedated with $0.5 \mathrm{mg} / \mathrm{g}$ Avertin $(1 \mathrm{~g}$ 2,2,2-tribromo

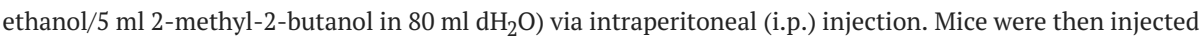
with $50 \mu \mathrm{l}$ in vivo D-luciferin (i.p.; $30 \mathrm{mg} / \mathrm{ml}$ in PBS; Gold Biotechnology, St. Louis, MO). At 10 min postluciferin, mice were imaged with the exposure time set to five minutes. Imaging elucidated six lines of mice with quantifiable photon emission (Fig. 2). The offspring from these six lines were screened at four to seven weeks by bioluminescence imaging using the aforementioned procedure.

\section{Bioluminescence imaging}

All images were captured with an IVIS 50 Imaging System using a five minute exposure time. To quantify and localize photon emission in live, intact animals, mice were anesthetized by i.p. injection with avertin $(250 \mathrm{mg} / \mathrm{kg}$ ), then injected i.p. with the substrate for the luciferase enzyme (luciferin $150 \mathrm{ul} \mathrm{of} 28.5 \mathrm{mg} / \mathrm{ml}$ stock [Xenogen]). Fur was removed over the area of interest with clippers or Nair (Church \& Dwight Co, Princeton, NJ). For non-survival imaging studies involving dissection and brain removal, mice were anesthetized with an overdose $(2 \mathrm{ml} / \mathrm{kg}$, i.p.) of a ketamine/xylazine cocktail $(53.7 \mathrm{mg} / \mathrm{ml}$ ketamine and 9.26 $\mathrm{mg} / \mathrm{ml}$ xylazine). After anesthesia, mice were given a $150 \mu \mathrm{l}$ (screening studies) or $500 \mu \mathrm{l}$ (dissection studies) i.p. bolus of $28.5 \mathrm{mg} / \mathrm{ml}$ stock $D$-luciferin (Xenogen). At five minutes post-luciferin, mice were imaged in one of the following positions: full body dorsal, full body ventral, head dorsal, head ventral. In some cases, multiple images were captured sequentially to qualitatively assess the distribution of bioluminescence at different stages of dissection. All images were collected within one hour of luciferin injection and comparison images between lines were captured at the same timepoint after luciferin injection, usually in the same exposure. 
When appropriate, post mortem removal of the skin dorsal to the skull and the dorsal skull plates was rapidly performed to expose the surface of the brain. Following in situ imaging, the brain was removed and either reimaged, frozen over dry ice in Tissue-Tek O.C.T. (Ted Pella Inc, Redding, CA) and/or dissected as necessary. If dissected, coronal and parasagittal segments were cut to be $2-4 \mathrm{~mm}$ thick. Between each dissection step, images were captured; image start times ranged from 5-46 min post-luciferin injection.

After image capture, Xenogen Living Image software v.2.50.2 was used to visualize and quantify bioluminescence. Bioluminescence was measured in photons $/ \mathrm{sec} / \mathrm{cm}^{2} / \mathrm{steradian}\left(\mathrm{p} / \mathrm{sec} / \mathrm{cm}^{2} / \mathrm{sr}\right)$ within a defined region of interest (ROI). ROI ovals were placed so as to maximize the signal measured over the area desired.

\section{Microdissection and in vitro luminometry}

After anesthetic overdose, whole brains from sex and age-matched line 928, 887, wild type (WT) and cytomegalovirus promoter-controlled luciferase (CMV-Luc) transgenic mice were removed and immediately frozen at $-86^{\circ} \mathrm{C}$ in Tissue-Tek O.C.T. as described above. Frozen brains were later thawed and microdissected using a dissecting microscope to isolate $0.01-0.03 \mathrm{~cm}^{3}$ tissue samples; isolated tissue samples were subsequently refrozen at $-86^{\circ} \mathrm{C}$ in microcentrifuge tubes. Frozen tissue samples were thawed and 5:1 v:v of Glo Lysis Buffer (Promega, Madison, WI) was added to each tube. Immediately after addition of the Glo Lysis Buffer, the tissue samples were physically dissociated using a pipette for one minute and then incubated for five minute at RT. After incubation, the samples were subjected to one freeze-thaw cycle over dry ice, spun down, and the supernatant was isolated.

To analyze luciferase activity in brain areas of interest $20 \mathrm{ul}$ aliquots of tissue sample supernatant was combined with $20 \mathrm{ul}$ of in vitro Bright-Glo luciferin (Promega, Madison, WI) for $10 \mathrm{~min}$. Luminescence was measured for $10 \mathrm{~s}$ on a Lumat LB 950 luminometer (Berthold Technologies GmbH \& Co KG, Bad Wildbad, Germany). Bradford protein assays were performed on 10:290 dilutions of tissue sample supernatant aliquots using Coomassie Plus (Thermo Scientific, Rockford, IL). RLU per $\mu g$ of protein was then determined for each sample. All data points were included in analysis unless identified as outliers by Grubbs' test. Results were graphed as mean and SE of CMV-luc $(n=6)$, WT $(n=4)$, line $928(n=4)$ and line $887(n=2$ or $n=4$, depending on region; SE bars not included for regions where $n=2)$. When appropriate, statistical comparisons were performed using 2-tailed student's t-tests. A threshold for significance was set at $\mathrm{p}<0.05$.

\section{Results}

The aim of this study was to create a mouse exhibiting quantifiable bioluminescence in catecholaminergic neurons, including DA mesencephalic cells. Accordingly, TH-Luc mice were created by microinjection of the linear $\mathrm{p} \beta$-globin4.5TH-Luc plasmid fragment (Fig. 1) into fertilized FVB/N mouse oocyte nuclei. Six TH-Luc transgenic lines were obtained that exhibited quantifiable luciferase expression as adults (Fig. 2).

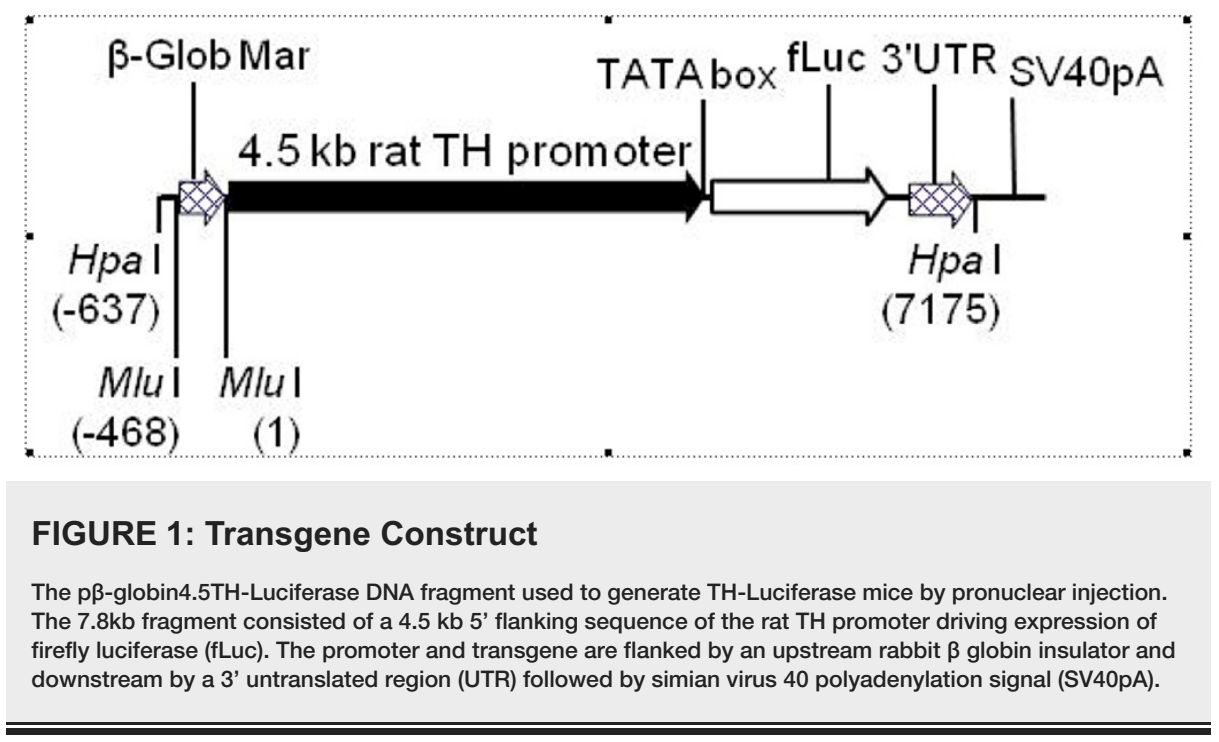




\section{Cureus}

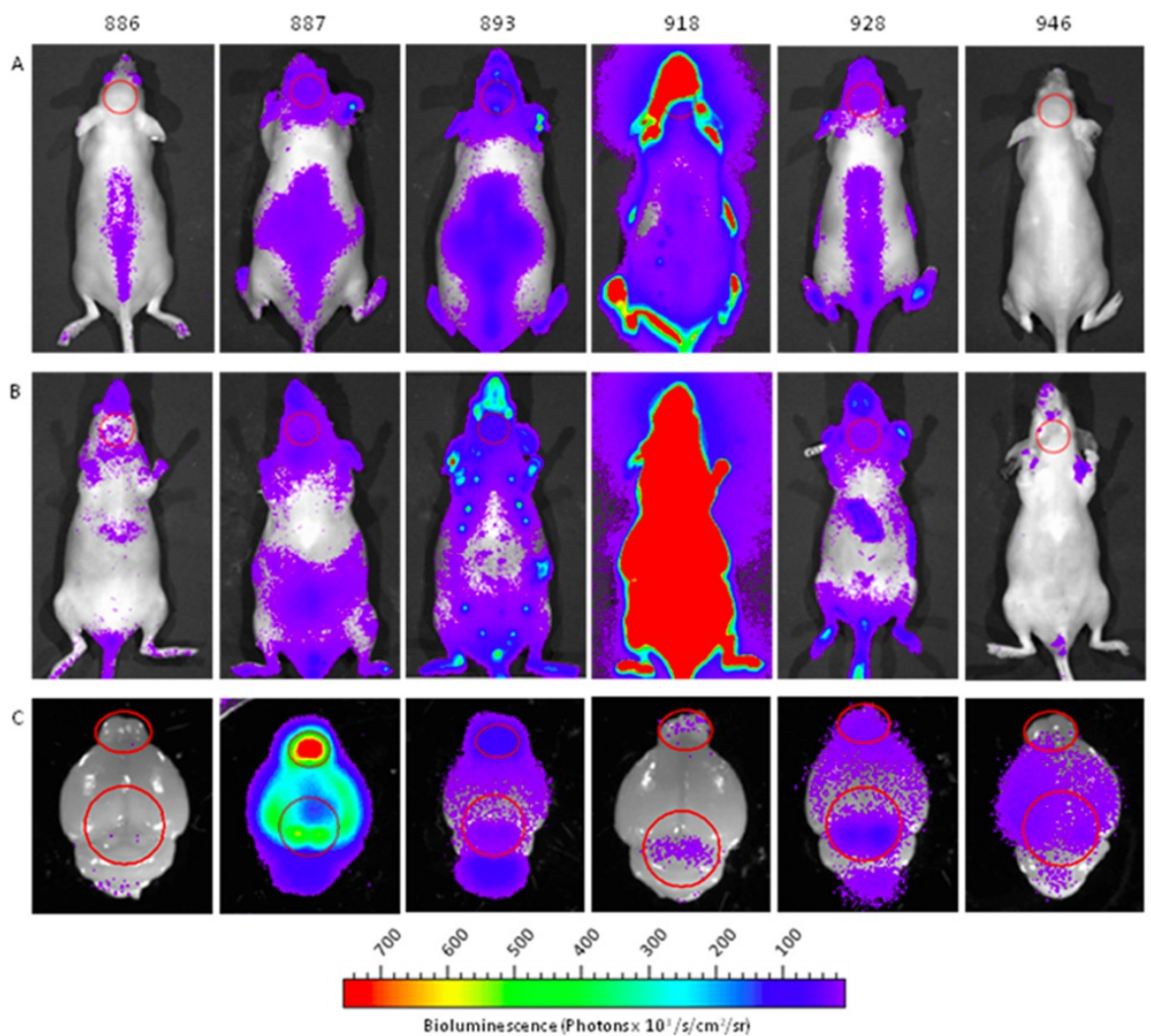

\section{FIGURE 2: Six TH-Luciferase positive founder lines}

Sequential imaging of whole mice dorsally (A: 5 min post-luciferin) and ventrally (B: 11 min post-luciferin), and of brains isolated from the same animals (C: $36 \mathrm{~min}$ post-luciferin); ROls ellipses enclose the area of highest bioluminescence over the skull (A, B: $3.14 \mathrm{~cm} 2)$, caudal brain (C: $1.02 \mathrm{~cm} 2)$, and olfactory bulbs (C: $0.41 \mathrm{~cm} 2)$.

\section{Analysis of gross TH-Luc expression patterns}

Whole-body imaging of the TH-Luc mice revealed luciferase expression over the vertebral column, eyes, rostral skull, caudal skull, and snout in most but not all of the six selected lines (Fig. 2A). When mice were imaged ventrally, bioluminescence was detected over the snout and anogenital area in all lines (Fig. 2B).

Bioluminescence on the dorsal brain surface of whole brains (Fig. 2C, 3B) was concentrated over the olfactory bulbs (OB) in lines $887,893,918$, the ventral midbrain (VM) in $887,893,918$, and 928 , caudal brain stem in lines $887,893,928$, and a diffuse signal was also seen emanating from the cerebral cortex (CTX) in 887, 893, 928 and 946. No significant luciferase activity was evident upon imaging the brain of line 886. Line 918 was significant for much stronger external bioluminescence than brain bioluminescence. 


\section{Cureus}

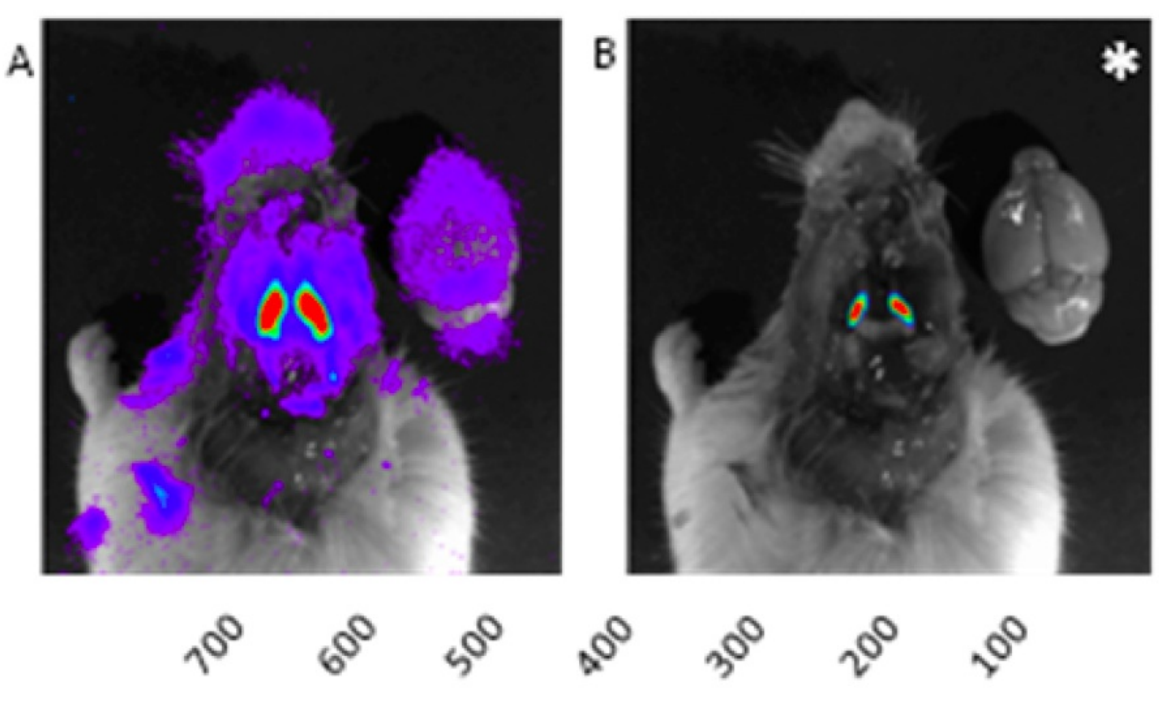

Bioluminescence (Photons $\times 10^{3} / \mathrm{s} / \mathrm{cm}^{2} / \mathrm{sr}$ )

\section{FIGURE 3: Cranial bioluminescence outside the brain}

Bioluminescence was detected in structures immediately ventral to the brain. Removal of the brain revealed dorsal brain expression consistent with previous experiments (A; 35 min post-luciferin) and bioluminescence in the trigeminal nerve (A, B; 35 min post-luciferin). The bioluminescence scale was adjusted in all images marked with $\left(^{*}\right)$ to facilitate anatomical localization.

Grossly, the highest midbrain luciferase expression levels appeared to be present in lines 928 and 887, which were thus selected for further analysis. The patterns of external luciferase activity were comparable in these lines, though line 887 exhibited greater overall levels of bioluminescence, with particularly strong bioluminescence over the $\mathrm{OB}$.

In dissection studies of animals from lines 928 and 887, incision and reflection of the scalp revealed a concentrated area of expression over the caudal skull (data not shown). To assess the possible contribution of extra-axial structures to overall bioluminescence, the cranial cavity was exposed by removal of the brain and dorsal skull plates. Dorsal imaging of the residual cranial cavity revealed a high level of luminescence in the trigeminal nerve (Fig. 3A, 3B). No other structures in the residual cranial cavity appeared to exhibit significant bioluminescence.

\section{Analysis of luciferase activity in the brains of TH-Luc mice}

For further localization of bioluminescence to specific neural structures, bioluminescence imaging following coronal (Fig. 4) and sagittal (Fig. 5) gross brain sectioning was performed in lines 928 and 887. Exact localization of structures in gross slices was necessarily limited by the imprecise nature of the gross slice procedures; shown labels in Figure 4 and Figure 5 are approximate. 


\section{Cureus}
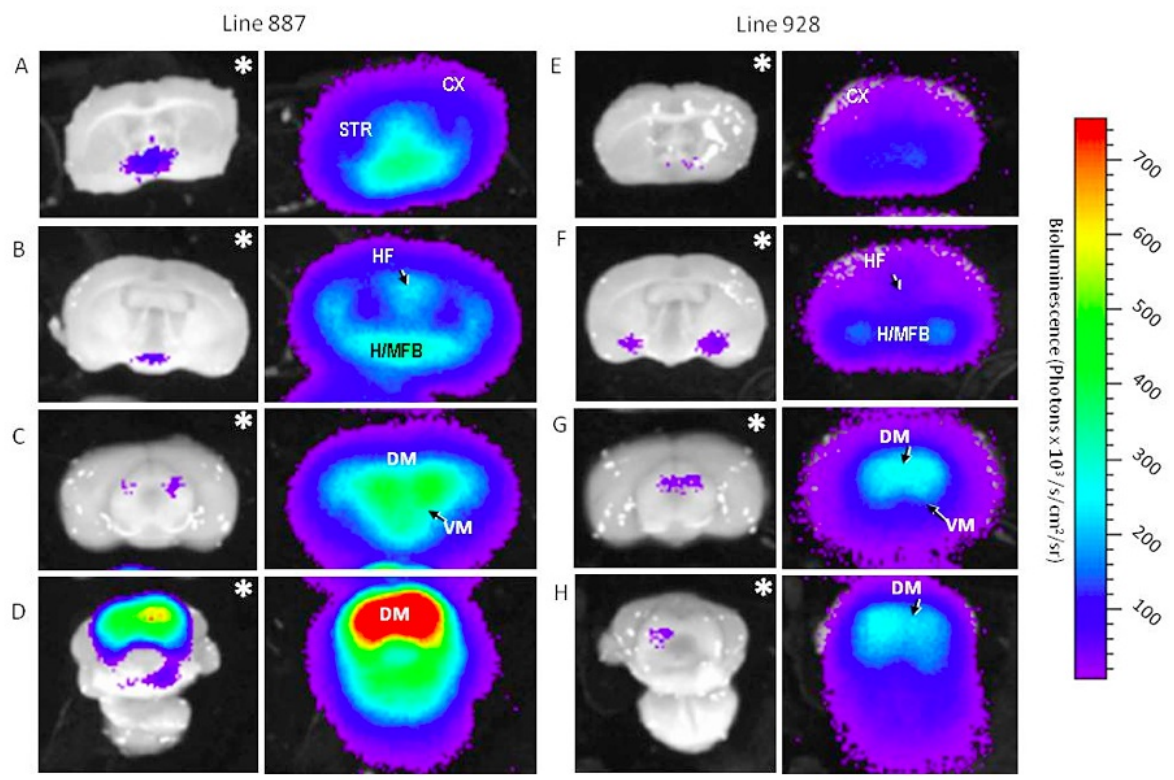

\section{FIGURE 4: Luciferase expression patterns in coronal brain slices}

From top to bottom, coronal slices were cut from one mouse of each line in a rostral to caudal manner (line 887: A-D; line 928: E-H). Bioluminescence identifies areas of luciferase activity. Approximate anatomical landmarks are indicated (H/MFB, hypothalamic area including medial forebrain bundle; VM, ventral midbrain; LC, locus ceruleus; DM, dorsal midbrain). The bioluminescence scale was adjusted in all images marked with $\left.{ }^{*}\right)$ to facilitate anatomical localization. Images were captured at $30 \min (C, D, G, H)$ or $36 \min (A, B, E, F)$ postluciferin administration. 


\section{Cureus}
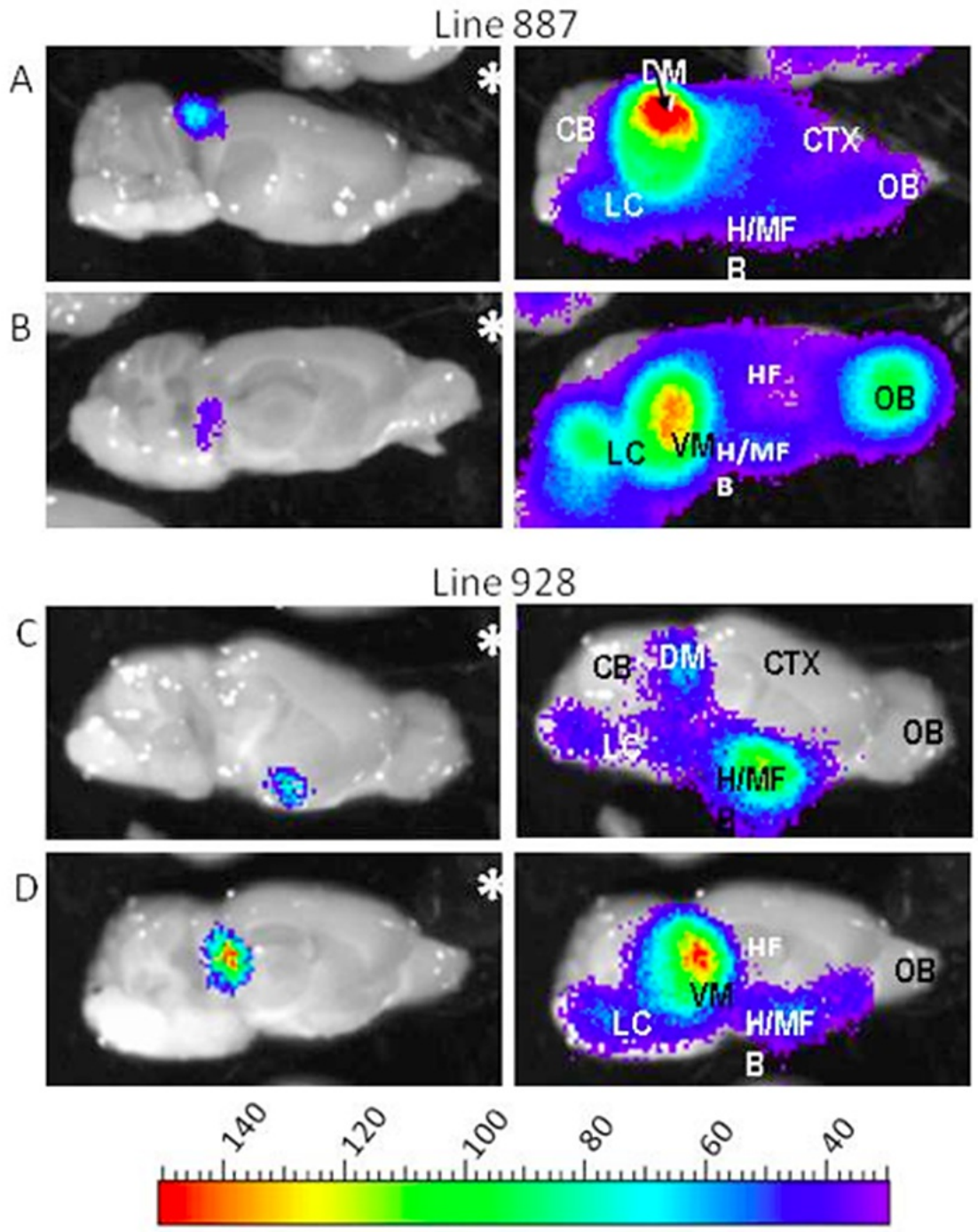

Bioluminescence (Photons $\times 10^{3} / \mathrm{s} / \mathrm{cm}^{2} / \mathrm{sr}$ )

\section{FIGURE 5: Luciferase activity patterns in parasagittal brain slices}

TH-Luciferase transgenic mice (line 887: A, B; line 928 C, D). The bioluminescence scale was adjusted in all images marked with $\left(^{*}\right)$ to facilitate anatomical localization. Images were captured 46 min post-luciferin administration.

Taken together, however, imaging of these gross slices were notable for particularly robust expression in the dorsal midbrain (DM). VM expression was evident at a lower level. OB expression, while robust in line 887, appeared less prominent in line 928. In vitro luminometry was additionally performed following microdissection of frozen brain samples (Fig. 6). 

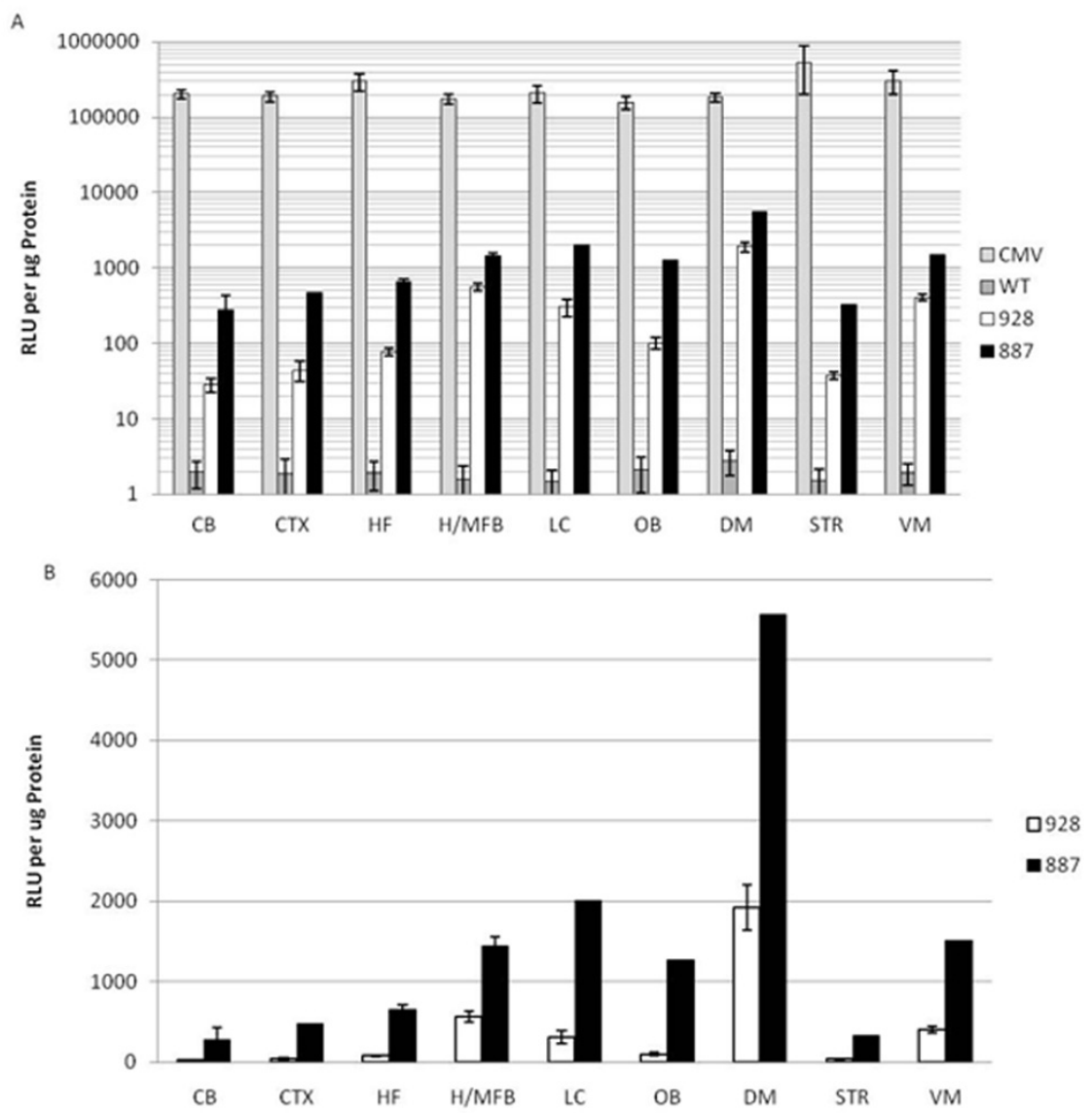

FIGURE 6: Quantification of luminescence from microdissected samples

Microdissected brain regions of WT, CMV-Luc, and TH-Luc lines 928, and 887 mice plotted on a semi-log (A), or linear (B). Tissue samples were isolated from areas corresponding to those indicated in figures 4 and 5 .

Brains were halved, and microdissected samples were obtained from each hemisphere, permitting two samples to be obtained from each mouse for each location. Sample numbers were: WT $(n=4)$, CMV-luc $(n=6)$, line 887 ( $n=2$ or 4, depending on area), and line $928(n=6)$. Locations examined included cerebellum (CB), hippocampal formation (HF), hypothalamus (including the medial forebrain bundle; H/MFB), locus ceruleus (LC), striatum (STR), OB, DM (including the superior and inferior colliculi), and VM (including the substantia nigra and ventral tegmental area). No differences were observed in levels of luciferase activity between areas in WT mice or in CMV-luc mice. Overall, measured bioluminescence levels were five-logs higher in samples from CMV-luc than WT mice in examined regions. Bioluminescence measured from microdissected samples of TH-luc lines 928 and 887 fell roughly midway between CMV-luc and WT mice when depicted on a log plot (Fig. 6A).

To facilitate visualization of measured difference between areas in lines 887 and 928, a linear graph including only these two lines was also prepared (Fig. 6B). Results appeared to correlate appropriately with findings evident from slice imaging; revealing, for example, stronger expression in the DM than in any other region ( $\mathrm{p}<0.005$, line 887 ), and proportionately weaker OB expression compared to other areas in 928 than was the case for 887. For each area examined in which $n>3$ for both lines (CB, HF, and H/MFB), luminescence was significantly higher for 887 than $928(\mathrm{p}<0.05)$. The area of highest expression was the $\mathrm{DM}$, though this expression was approximately two logs lower than that of any region in CMV-luc mice, presumably consistent with the relatively low percentage of luciferase-expressing cells in the microdissected TH-luc samples, and the strong nature of the CMV promoter.

Strong expression was also observed in the H/MFB, LC, and VM, of both lines. Though luciferase activity in the OB for both lines exceeded that of other control areas, including CB, CTX, and HF, such activity in line 928 was proportionately weak compared to line 887 . STR, though not intended as a control area, proved to have among the lowest levels of luciferase activity. The levels of bioluminescence observed in the lowestexpressing areas of line 887 or 928 remained one to two logs higher than that observed in WT mice (Fig. 6A).

\section{Discussion}


Here we report the generation and preliminary characterization of transgenic TH-Luc mouse lines expressing firefly luciferase under control of the $4.5 \mathrm{~kb}$ rat TH promoter. Six lines were identified as to both carry the transgene, and exhibit measurable levels of luciferase activity. The intensity and pattern of such activity varied widely from line 918 , with robust expression superficially, but relatively low expression intracranially, to line 887 , with highly robust intracranial expression relative to superficial expression. Two lines, 887 and 928, were selected for further analysis. Both exhibited markedly greater luciferase activity in all brain regions examined, compared to WT mice, with prominent expression in known areas of TH expression, including VM, OB, LC, and H/MFB. However, the relative OB luciferase activity appeared stronger in line 887 than 928. In both lines, luciferase activity was about two-fold higher in the DM than in any other brain region, even though TH expression is not known to occur in the DM of adult animals.

Variability in the intensity of transgene expression between animals can likely be accounted for by varying numbers of transgene copies leading to overall higher levels of expression in some animals than others, in addition to differences in the location of construct integration, with those generated via integration in more open chromatin exhibiting generally higher levels of expression. The marked differences in patterns of expression between animals of different lines is perhaps also best explained by differences in location of integration, with functional enhancers for different patterns of either appropriate (VM, OB, or cutaneous autonomic and/or sensory fibers) being differentially available at the integration sites achieved in the different lines.

Upon imaging of the dorsal brain, at least four of the lines demonstrated a focus of expression over the DM, suggesting this to be a reproducible pattern resulting from use of the $4.5 \mathrm{~kb}$ TH promoter. TH expression occurs in the tectum during development, with this region of the DM subsequently giving rise to the adult superior and inferior colliculus. It would seem reasonable to speculate that a region of the full TH promoter responsible for inhibition of TH expression in the adult DM is absent from the $4.5 \mathrm{~kb}$ TH promoter. Indeed, similar findings have been noted in prior publications of reporter genes driven by the $4.5 \mathrm{~kb} \mathrm{TH}$ promoter [14, 17-22].

That much higher than WT levels of expression were observed in essentially non-dopaminergic brain regions, such as the CTX and CB, may additionally argue for the failure of appropriate inhibition of expression using the truncated promoter. Certain dopaminergic neurons from the ventral tegmental area are known to project to the cortex, while certain cell populations in the CB transiently express dopamine during embryogenesis; however, a much lower ratio of TH expression would be expected in these adult regions than is observed in the TH-Luc lines 928 and 887.

Somewhat surprisingly, the level of luciferase expression was quite low in the STR, the projection area for dopaminergic neurons of the substantia nigra. These nigrostriatal projections represent the ones that degenerate in patients with PD leading to impairments in motor function. Quite strong levels of luciferase activity were observed in the VM. As such, the lack of striatal luminescence could be explained by such VM activity arising exclusively from the ventral tegmental area, with inappropriate lack of luciferase expression in the SNc. Though subregion microdissections were not attempted to exclude this possibility, we consider it more likely that luciferase is synthesized in the soma of nigrostriatal neurons, and that lack of active axonal transport of luciferase, combined with rapid degradation of the labile protein at physiologic temperatures, prevent active luciferase protein from diffusing far enough along nigrostrial projections to reach appreciable levels in the STR. An available antibody against luciferase would facilitate further investigation of this question; however, of all the commercially available luciferase antibodies that we, and others we consulted with have tried, none have yielded adequate performance for application in IHC assays.

The TH-luc mice described herein were developed in part to assess the feasibility of imaging neuronal subpopulations over time in living animals. While several groups have demonstrated the feasibility of intracranial monitoring of luciferase-expressing tumors, very large numbers of strongly expressing cells in these models may help surmount any normal difficulties in the visualization of intracranial bioluminescence. Many fold higher luciferase expression was present in the trigeminal nerve than in even the DM of line 928 (Fig. 3). Such expression, however, could be rendered unnoticeable under the normally situated brain due to the light-scattering property of normal brain tissue [23]. Certainly, the presence of strong DM expression in the presently described mice would preclude meaningful monitoring of luciferase activity from any deeper structures.

Longer fragments of the TH promoter have been noted to enhance the specificity of reporter genes [7, 24]. Additionally, a knock-in mouse with the dopamine transport (DAT) linked to the enzyme cre recombinase has recently been reported to have excellent specificity to dopaminergic neurons [25]. Development of mice expressing firefly luciferase under such promoters may represent logical next steps to more critically assess the feasibility of in vivo visualization of DA neurons in living mice.

\section{Conclusions}

In sum, these TH-Luc mice represent the first step towards developing a strategy for in vivo imaging of DA neurons. Future generations of such mice, which would ideally be marked by decreased an absence of DM transgene expression, may have applications in the study of SNc DA cell degradation in PD facilitating the 
future investigations into therapies to halt DA cell loss. DA-bioluminescent mice, if successful, could serve not only as valuable models for high throughput screening of neuroprotecitve compounds in lesion paradigms, but also as a source of stem cells or primary catecholaminergic neurons for in vitro toxopharmacology studies or in vivo transplantation studies.

\section{Additional Information \\ Disclosures}

Human subjects: All authors have confirmed that this study did not involve human participants or tissue. Animal subjects: The University of Minnesota Institutional Animal Care and Use Committee Issued protocol number N/A. Conflicts of interest: In compliance with the ICMJE uniform disclosure form, all authors declare the following: Payment/services info: This work was supported in part by funds from the Ben Miller Foundation, E. Isaksen Parkinson's Disease Research Fund, D. Cavin Memorial Fund, R.C. Cornford and V. Cornford Endowment Fund, Turner Family Fund, NIH Medical Scientist Training Program, University of Minnesota Undergraduate Research Opportunity Program and NIH R03-NS060059. Financial relationships: All authors have declared that they have no financial relationships at present or within the previous three years with any organizations that might have an interest in the submitted work. Other relationships: All authors have declared that there are no other relationships or activities that could appear to have influenced the submitted work.

\section{Acknowledgements}

The authors thank Dr. Dona M. Chikaraishi of Duke University for the p4.5TH-Luc plasmid, the University of Minnesota Mouse Genetics Laboratory for performing the pronuclear injections, Seung Uk Oh for his assistance with PCR, and Brian M. Anderson for his help screening TH-Luc offspring. We also thank Erin M. Broberg for her assistance in drafting the manuscript and figures therein, and Dr. R. Scott McIvor for his critical reading of early drafts.

\section{References}

1. Cotzias GC, Van Woert MH, Schiffer LM: Aromatic amino acids and modification of parkinsonism. N Engl J Med . 1967, 276:374-79.

2. Benabid AL, Pollak P, Seigneuret E, Hoffmann D, Gay E, Perret J: Chronic VIM thalamic stimulation in Parkinson's disease essential tremor and extra-pyramidal dyskinesias. Acta Neurochir. 1993, 58:39-44.

3. Beal MF: Experimental models of Parkinson's disease. Nat Rev Neurosci. 2001, 5:325-34.

4. Fleming SM, Chesselet MF: Behavioral phenotypes and pharmacology in genetic mouse models of Parkinsonism. Behav Pharmacol. 2006, 17:383-91.

5. Emborg ME: Evaluation of animal models of Parkinson's disease for neuroprotective strategies. J Neurosci Methods . 2004, 139:121-43.

6. Mandel S, Grunblatt E, Riederer P, Gerlach M, Levites Y, Youdim MB: Neuroprotective strategies in Parkinson's disease: an update on progress.. CNS Drugs. 2003, 17:729-62.

7. Sawamoto K, Nakao N, Kobayashi K, Matsushita N, Takahashi H, Kakishita K, Yamamoto A, Yoshizaki T, Terashima T, Murakami F, Itakura T, Okano H: Visualization direct isolation and transplantation of midbrain dopaminergic neurons. Proc Natl Acad Sci USA. 2001, 98:6423-28.

8. Drucker-Colin R, Verdugo-Diaz L: Cell transplantation for Parkinson's disease: present status . Cell Mol Neurobiol . 2004, 24:301-16

9. Michaeli S, Burns TC, Kudishevich E, Harel N, Hanson T, Sorce DJ, Garwood M, Low WC: Detection of neuronal loss using $\mathrm{T}(1 \mathrm{rho}) \mathrm{MRI}$ assessment of (1) $\mathrm{H}(2) \mathrm{O}$ spin dynamics in the aphakia mouse. J Neurosci Methods . 2009, 117:160-67.

10. Strome EM, Doudet DJ: Animal models of neurodegenerative disease: insights from in vivo imaging studies . Mol Imaging Biol . 2007, 9:186-95.

11. Chatziioannou AF: Molecular imaging of small animals with dedicated PET tomographs . Eur J Nucl Med Mol Imaging . 2002, 29:98-114.

12. Sadikot RT, Blackwell TS: Bioluminescence imaging. Proc Am Thorac Soc . 2005, 2:537-40.

13. Welsh DK, Kay SA: Bioluminescence imaging in living organisms. Curr Opin Biotechnol. 2005, 16:73-78.

14. Liu J, Merlie JP, Todd RD, O'Malley KL: Identification of cell type-specific promoter elements associated with the rat tyrosine hydroxylase gene using transgenic founder analysis. Brain Res Mol Brain Res. 1997, 50:33-42.

15. Deroose CM, Reumers V, Gijsbers R, Bormans G, Debyser Z, Mortelmans L, Baekelandt V: Noninvasive monitoring of long-term lentiviral vector-mediated gene expression in rodent brain with bioluminescence imaging. Mol Ther . 2006, 14:423-31.

16. Ohlfest JR, Demorest ZL, Motooka Y, Vengco I, Oh S, Chen E, Scappaticci FA, Saplis RJ, Ekker SC, Low WC, Freese $\mathrm{AB}$, Largaespada DA: Combinatorial antiangiogenic gene therapy by nonviral gene transfer using the sleeping beauty transposon causes tumor regression and improves survival in mice bearing intracranial human glioblastoma. Mol Ther . 2005, 12:778-88.

17. Schimmel JJ, Crews L, Roffler-Tarlov S, Chikaraishi DM: $4.5 \mathrm{~Kb}$ of the Rat Tyrosine Hydroxylase 5' Flanking Sequence Directs Tissue Specific Expression during Development and Contains Consensus Sites for Multiple Transcription Factors.. Brain Res Mol Brain Res . 1999, 74:1-14.

18. Banerjee SA, Hoppe P, Brilliant M, Chikaraishi DM: 5' Flanking Sequences of the Rat Tyrosine Hydroxylase Gene Target Accurate Tissue-Specific Developmental and Transsynaptic Expression in Transgenic Mice. J Neurosci . 1992, 12:4460-67.

19. Gelman DM, Noain D, Avale ME, Otero V, Low MJ, Rubinstein M: Transgenic mice engineered to target 


\section{Cureus}

Cre/loxP-mediated DNA recombination into catecholaminergic neurons. Genesis . 2003, 36:196-202.

20. Kessler MA, Yang M, Gollomp KL, Jin H, Iacovitti L: The human tyrosine hydroxylase gene promoter. Brain Res Mol Brain Res. 2003, 112:8-23.

21. Nagatsu I, Karasawa N, Yamada K, Sakai M, Fujii T, Takeuchi T, Arai R, Kobayashi K, Nagatsu T: Expression of human tyrosine hydroxylase-chloramphenicol acetyltransferase (CAT) fusion gene in the brains of transgenic mice as examined by CAT immunocytochemistry. J Neural Transm Gen Sect . 1994, 96:85-104.

22. Trocme C, Sarkis C, Hermel JM, Duchateau R, Harrison S, Simonneau M, Al-Shawi R, Mallet J: CRE and TRE sequences of the rat tyrosine hydroxylase promoter are required for TH basal expression in adult mice but not in the embryo. Eur J Neurosci . 1998, 10:508-21.

23. Björkund A, Hökfelt T: Classical transmitters in the CNS. Vol. 2 . Elsevier Science Publishers, Amsterdam; 1984.

24. Matsushita N, Okada H, Yasoshima Y, Takahashi K, Kiuchi K, Kobayashi K: Dynamics of tyrosine hydroxylase promoter activity during midbrain dopaminergic neuron development. J Neurochem. 2002, 82:295-304.

25. Zhuang X, Masson J, Gingrich JA, Rayport S, Hen R: Targeted gene expression in dopamine and serotonin neurons of the mouse brain. J Neurosci Methods. 2005, 143:27-32. 